\title{
A potential link between polycystic ovary syndrome and non-alcoholic fatty liver disease: an update meta-analysis
}

\author{
Jia $\mathrm{Wu}^{1+}$, Xin-Yu Yao ${ }^{2 \dagger}, \mathrm{Ru}-\mathrm{Xia}$ Shi ${ }^{1}$, Su-Fen Liu ${ }^{1}$ and Xiao-Yong Wang ${ }^{2 *}$
}

\begin{abstract}
Background: Epidemiological literature regarding the effect of polycystic ovary syndrome (PCOS) as a risk factor for non-alcoholic fatty liver disease (NAFLD) remains inconsistent. Furthermore, it remains debatable whether NAFLD is associated with PCOS as a consequence of shared risk factors or whether PCOS contributes to NAFLD in an independent fashion. Therefore, this meta-analysis was conducted.

Methods: This meta-analysis was conducted in accordance with the guidelines of the Preferred Reporting Items for Systematic Reviews and Meta-Analyses (PRISMA). Relevant studies published before May 2017 were identified and retrieved from PubMed and Web of Science databases. The data were extracted, and the pooled odds ratios (ORs) and 95\% confidence intervals (95\% Cls) were calculated.
\end{abstract}

Results: A total of 17 studies were included into the present analysis. Compared to the control group, the risk of NAFLD in the PCOS group was higher $(\mathrm{OR}=2.25,95 \% \mathrm{Cl}=1.95-2.60)$. When stratified by $\mathrm{BMI}$ and geographic location, the results indicated that the frequency of NAFLD risk was significantly higher in obese subjects ( $\mathrm{OR}=3.01,95 \% \mathrm{Cl}=1.88-4.82)$, non-obese subjects ( $\mathrm{OR}=2.07,95 \% \mathrm{Cl}=1.12-3.85)$, subjects from Europe $(\mathrm{OR}=2.00,95 \% \mathrm{Cl}=1.58-2.52)$, subjects from the Asia-Pacific Region, $(\mathrm{OR}=2.32,95 \% \mathrm{Cl}=1.89-2.84)$ and subjects from America $(\mathrm{OR}=2.96,95 \% \mathrm{Cl}=1.93-4.55)$. In addition, PCOS patients with hyperandrogenism (HA) had a significantly higher risk of NAFLD, compared with controls $(\mathrm{OR}=3.31,95 \% \mathrm{Cl}=2.58-4.24)$. However, there was no association between PCOS patients without HA and higher risk of NAFLD ( $\mathrm{OR}=1.46 ; 95 \% \mathrm{Cl}=0.55-3.87)$. The results of this meta-analysis should be interpreted with caution due to the small number of observational studies and possible confounding factors.

Conclusion: The meta-analysis results suggest that PCOS is significantly associated with high risk of NAFLD. Although this association was independent of obesity and geographic region, it might be correlated with HA.

Keywords: Polycystic ovary syndrome, Non-alcoholic fatty liver disease, Obesity, Hyperandrogenism, Meta-analysis

\section{Plain English summary}

At present, a question that should be addressed is whether non-alcoholic fatty liver disease (NAFLD) is associated with polycystic ovary syndrome (PCOS) as a consequence of shared risk factors, or whether PCOS contributes to NAFLD in an independent fashion.

In the present study, a meta-analysis was performed to shed some light into this issue.

\footnotetext{
* Correspondence: wxy20009@126.com

${ }^{\dagger}$ Equal contributors

2Department of Gastroenterology, Changzhou No. 2 Hospital, Affiliated with

Nanjing Medical University, Changzhou 213000, China

Full list of author information is available at the end of the article
}

A total of 17 studies were included into the metaanalysis. PCOS was significantly associated with higher risk of NAFLD, with an approximately 2.3-fold higher chance. This association was independent of obesity and geographic region but might be correlated with hyperandrogenism.

In conclusion, the screening for NAFLD is only needed for hyperandrogenic women with PCOS, and not for all women with PCOS.

\section{Background}

Non-alcoholic fatty liver disease (NAFLD) is an increasingly common form of chronic disorder in the Western world, and is characterized by fat accumulation in the 
liver, which is independent of significant alcohol consumption, the use of steatogenic medications, or hereditary disorders [1, 2]. NAFLD comprises of a wide spectrum of liver diseases that range from simple steatosis to nonalcoholic steatohepatitis (NASH), leading to cirrhosis [2]. Furthermore, NAFLD prevalence markedly increases with age [3]. The estimated prevalence of NAFLD in the general population ranges within $6.3-33 \%$, with a median of $20 \%$ [2]. However, in obese or type-2 diabetes mellitus (T2DM) patients, the prevalence of NAFLD can increase to approximately $75 \%$ [4]. Most patients with NAFLD remain asymptomatic, although it can slowly progress to cirrhosis, thereby increasing the risk of hepatocellular carcinoma (HCC) [5]. NAFLD is also the most common cause of cryptogenic cirrhosis [6]. Given its high prevalence and the fact that NAFLD is becoming a major public health hazard, it is very important to unveil its potential risk factors, since early treatment can prevent the progression of liver disease. Present evidence strongly indicates that obesity, T2DM, unhealthy lifestyle, dyslipidemia, male gender and ethnicity are risk factors for NAFLD development [2,3]. Since most patients with NAFLD have insulin resistance (IR), the association between NAFLD and IR has been widely accepted [7]. In fact, the role of IR in the pathogenesis of NAFLD has been thoroughly studied, and a strong association has been shown $[8,9]$.

Another disorder associated with IR is polycystic ovary syndrome (PCOS). PCOS is an exceptionally common endocrine disorder in premenopausal women of reproductive age. It is characterized by hyperandrogenism (HA), chronic anovulation and polycystic ovaries (PCO) through ovarian ultrasonography, when other etiologies are excluded $[10,11]$. It is possible that the link between PCOS and NAFLD might be IR. Since the first association between NAFLD and PCOS was reported in 2005 [12], many subsequent studies have investigated the association between PCOS and risk of NAFLD. Some authors have reported that patients with PCOS have a higher prevalence of NAFLD than controls [13-15]. However, other studies failed to establish a relationship between PCOS and the increased incidence of NAFLD $[16,17]$. Epidemiological literature regarding the effect of PCOS as a risk factor for NAFLD development remains inconsistent and inconclusive. A meta-analysis that comprised of data until June 2013 was performed by Ramezani-Binabaj et al. They reported that (1) the prevalence of NAFLD noticeably increased in women with PCOS, and (2) PCOS may be a significant risk factor for the development of NAFLD [18]. However, the metaanalysis was limited to only seven studies, and did not include stratified analyses. PCOS itself accounts for a higher risk of NAFLD. However, other specific factors such as androgen levels or body mass index (BMI) may contribute to NAFLD development in women with PCOS. Since 2013, many studies with larger sample sizes have reported information on factors such as obesity and androgen levels $[13,14]$.

Therefore, an update meta-analysis was conducted to investigate the association between PCOS and the risk of NAFLD development through studies published until May 2017. In order to explore whether NAFLD is associated with PCOS as a consequence of shared risk factors, or whether PCOS contributes to NAFLD in an independent fashion, stratified analyses were performed. Furthermore, factors associated with the presence of NAFLD in PCOS women were identified, and evidence of characteristics that can help identify women who have high risk of NAFLD were provided.

\section{Methods}

This meta-analysis was conducted in accordance with the guidelines of the Preferred Reporting Items for Systematic Reviews and Meta-Analyses (PRISMA) [19]. The research question was formulated according to the PICO model. P [population]: studies that recruited female patients as participants; I [Intervention or Exposure]: PCOS diagnosis; $\mathrm{C}$ [comparator]: PCOS group compared with the control group; O [outcome]: NAFLD.

\section{Literature search}

A comprehensive search was performed on PubMed and Web of Science databases for relevant published articles and abstracts on the association between PCOS and NAFLD published until May 2017. Merely studies performed in humans and those reported in the English language were considered. For PCOS, the following search terms were used: "PCOS, polycystic ovary syndrome, polycystic ovary". These terms were combined with the following terms for NAFLD: "NAFLD, Non-alcoholic fatty liver disease, NASH, non-alcoholic steatotic hepatitis, hepatic steatosis". For the association between PCOS and NAFLD risk, "risk, risk factor" was used.

Two independent reviewers (R-X.S. and J.W.) completed the initial screening process using pre-defined inclusion criteria (as detailed below). Articles were included for further review when these were original studies with primary data, involved human subjects, and were related to the topic of interest. These search results were independently reviewed by two reviewers and were compared for discrepancies. A third reviewer (X-Y.Y.) was involved to resolve any discrepancies that may occur between the two reviewers. In addition, the reference list of selected studies was manually reviewed for additional relevant publications.

\section{Inclusion criteria}

Studies were included in the meta-analysis when the following criteria were met: case-control studies, cohort studies and cross-sectional studies; studies that compared the 
prevalence of NAFLD in women with PCOS with matched controls; studies that contained sufficient data for analyses; studies published in the English language. For articles with overlapping data of the same population source, only the largest report was included.

\section{Exclusion criteria}

Articles were excluded when any of the following criteria were met: (1) review articles, meta-analyses, letters, commentaries, or case reports; (2) duplicates or continued work of previous publications; (3) studies without complete data; (4) articles that lack a control group; (5) articles not published in the English language. Studies without complete data, lack a control group and have overlapping data of the same population source were the common characteristics of the excluded papers.

\section{Data extraction and quality assessment}

Data extracted from each study included the following: name of the first author(s), year of publication, geographic region from which the study population was derived, diagnostic criteria for PCOS and NAFLD, and the sample size. The baseline information and data were independently extracted by the two primary reviewers (X-Y.W. and J.W.) using the same standard. The results were compared, and disagreements were resolved by consensus.

The quality of the included studies was independently assessed by two primary investigators (X-Y.W. and J.W.) using the Newcastle-Ottawa Scale (NOS) [20]. Each study was assessed based on three broad perspectives: selection, comparability and exposure/outcome. The score ranged within 0-9. Discrepancies were resolved by discussion through a third author (X-Y.Y.).

\section{Statistical analysis}

The association between the prevalence of NAFLD in women with PCOS and matched controls was calculated using odds ratios (ORs) and 95\% confidence intervals (CIs). In addition to the overall comparisons, subgroup analyses were performed based on geographic region, BMI, diagnostic criteria for PCOS and NAFLD, and androgen levels from articles with available adequate data. Heterogeneity between studies was assessed by Q-test and $I^{2}$-test. Heterogeneity was considered statistically significant at $P<0.05$ or $I^{2}>50 \%$, and a random-effects model was applied.

In the assessment for publication bias, Begg's test [21] and Egger's test were used [22]. If publication bias was detected, Duval and Tweedie's trim and fill method was used to adjust the results [23]. In addition, a sensitivity analysis was performed using the leave-one-out analysis. Statistical analyses were conducted using Stata software version 12 (StataCorp, College Station, TX, USA). $P<0.05$ was considered statistically significant.

\section{Results}

Summary of the literature search

A flow chart depicting the criteria for selecting studies for the present meta-analysis is shown in Fig. 1. In the first search, a total of 221 publications were initially retrieved. Among these publications, 64 were excluded due to duplication. After reviewing the remaining 157 abstracts, 137 publications that did not meet the predefined inclusion criteria stated in the Methods section were excluded. Furthermore, three studies were excluded after reviewing the full texts due to insufficient data [24-26]. Finally, 17 studies were included for analysis [13-17, 27-38] (Table 1). The NOS scale was used to assess the quality of studies included through the two independent reviewers. The NOS results revealed that the average quality score was 5.2 (range: 4-6; Table 1).

\section{Data analysis}

A total of 17 studies that compared women with PCOS with matched controls with regard to NAFLD risk were included. There was no significant heterogeneity in the included studies $\left(I^{2}=4.6 \%, P\right.$-heterogeneity $\left.=0.40\right)$. Therefore, the fixed-effects model was used for the meta-analysis. Compared to the control group, the PCOS group had a significantly higher risk of NAFLD $(\mathrm{OR}=2.25,95 \% \mathrm{CI}=1.95-2.60)$ (Fig. 2).

Subgroup analyses were also performed based on geographic region, BMI, diagnostic criteria for PCOS or NAFLD, and androgen levels. When stratified by geographic location, a significantly increased risk for developing PCOS-associated NAFLD was found in Europe $(\mathrm{OR}=2.00,95 \% \mathrm{CI}=1.58-2.52$; Fig. $3 \mathrm{a})$, the Asia-Pacific Region, $(\mathrm{OR}=2.32,95 \% \mathrm{CI}=1.89-2.84$; Fig. $3 \mathrm{~b})$ and America $(\mathrm{OR}=2.96,95 \% \mathrm{CI}=1.93-4.55$; Fig. 3c). Thus, these results strongly support that PCOS is associated with NAFLD risk, but is independent of geographic region.

According to the subgroup analyses based on the diagnostic criteria for PCOS, the effects of PCOS on NAFLD risk did not significantly vary when either the Rotterdam criteria $(\mathrm{OR}=2.19,95 \% \mathrm{CI}=1.88-2.55$; Fig. $4 \mathrm{a})$, or other criteria $(\mathrm{OR}=3.31,95 \% \mathrm{CI}=1.89-5.82$; Fig. $4 \mathrm{~b})$ was used, including the National Institute of Health (NIH) criteria and the Androgen Excess Society criteria. When stratified by the diagnostic method for NAFLD using ultrasonography, it was also found that PCOS was associated with NAFLD risk (OR $=2.29,95 \% \mathrm{CI}=1.96-2.67$; Fig. 4c).

When stratified by BMI, in which non-obese subjects were defined as BMI $<25 \mathrm{~kg} / \mathrm{m}^{2}$, a significantly increased prevalence for NAFLD risk was found to be associated with PCOS. This was found not only in obese subjects $(\mathrm{OR}=3.01,95 \% \mathrm{CI}=1.88-4.82$; Fig. $5 \mathrm{a})$, but also in nonobese subjects $(\mathrm{OR}=2.07,95 \% \mathrm{CI}=1.12-3.85$; Fig. $5 \mathrm{~b})$. Many studies have reported on the increased prevalence of NAFLD in women with PCOS, and this was most likely 


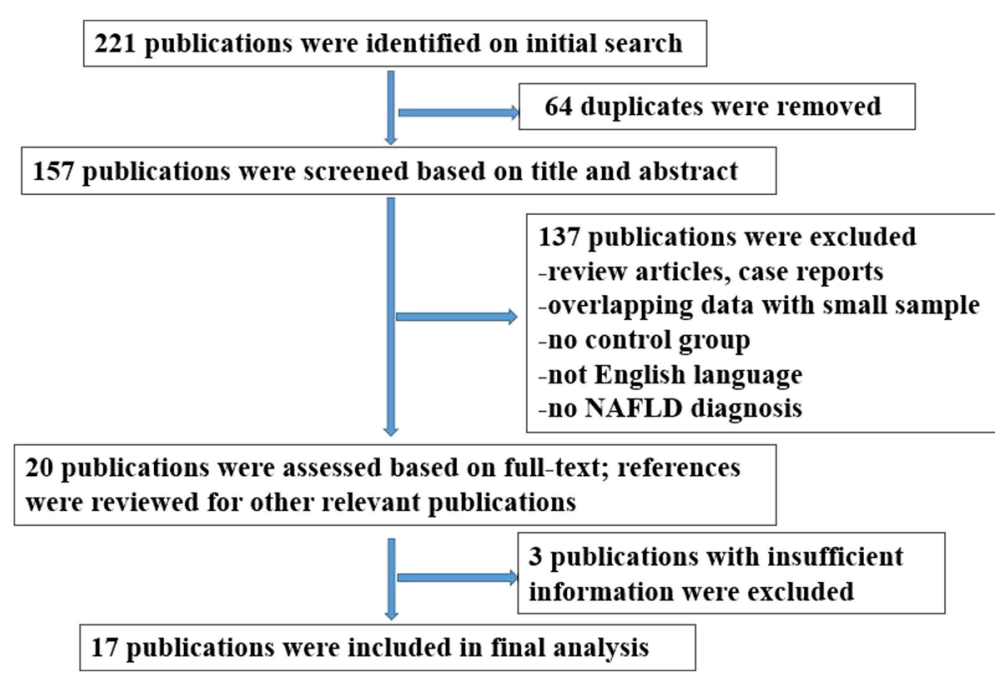

Fig. 1 Flow diagram of the study screening and selection process

due to obesity and IR, as previously suggested. The present data indicates that women with PCOS have an increased prevalence of NAFLD risk, which is independent of obesity.

According to subgroup analyses based on androgen levels, PCOS patients with HA have a significantly higher risk of NAFLD than controls $(\mathrm{OR}=3.31 ; 95 \% \mathrm{CI}=2.58-4.24$; Fig. 6a). However, in non-HA PCOS women, this was not associated with increased NAFLD risk when compared to controls $(\mathrm{OR}=1.46,95 \% \mathrm{CI}=0.55-3.87$; Fig. $6 \mathrm{~b})$. The results of the present study reveal that PCOS per se is not a risk factor that leads to the development of NAFLD, and $\mathrm{HA}$ is a contributing factor to the prevalence of NAFLD in women with PCOS.

Overall, no evidence of heterogeneity was found in the included studies when stratified by geographic region,

Table 1 Characteristics of the studies included in the meta-analysis

\begin{tabular}{|c|c|c|c|c|c|c|c|}
\hline First author & Year & Country & NAFLD criteria & PCOS criteria & PCOS (NAFLD) & Control (NAFLD) & $\operatorname{NOS}^{b}$ \\
\hline Cerda C & 2007 & Chile & Ultrasound & Rotterdam & $41(17)$ & $31(6)$ & 5 \\
\hline Markou A & 2010 & Greece & Ultrasound & Rotterdam & $17(0)$ & $17(0)$ & 6 \\
\hline Gutierrez-Grobe Y & 2010 & Mexico & Ultrasound & Not mentioned & $50(31)$ & $147(62)$ & 4 \\
\hline Vassilatou E & 2010 & Greece & Ultrasound & AES & $57(21)$ & $60(12)$ & 5 \\
\hline Hossain N & 2011 & USA & Histology & Rotterdam & $25(11)$ & $25(5)$ & 5 \\
\hline Zueff LF & 2012 & Brazil & Ultrasound & Rotterdam & $45(33)$ & $45(21)$ & 5 \\
\hline Karoli R & 2013 & India & Ultrasound & Rotterdam & $54(36)$ & $55(14)$ & 5 \\
\hline Qu Z & 2013 & China & Ultrasound & Rotterdam & $602(198)$ & $588(109)$ & 5 \\
\hline Kahal H & 2014 & UK & Ultrasound & Rotterdam & $19(7)$ & $17(0)$ & 6 \\
\hline Bohdanowicz-Pawlak A & 2014 & Poland & Ultrasound & Rotterdam & $184(106)$ & $125(62)$ & 5 \\
\hline Kuliczkowska Plaksej J & 2014 & Poland & Ultrasound & Rotterdam & $173(92)$ & $125(40)$ & 5 \\
\hline Romanowski MD & 2015 & Brazil & Ultrasound & AES & $101(24)$ & $30(1)$ & 5 \\
\hline Vassilatou E & 2015 & Greece & Ultrasound & Rotterdam & $40(31)$ & $70(40)$ & 6 \\
\hline Macut D & 2016 & Greece Serbia & NAFLD-LFS & Rotterdam & $600(304)$ & $125(43)$ & 5 \\
\hline Ayonrinde OT & 2016 & Australia & Ultrasound & $\mathrm{NIH}$ & $32(12)$ & $167(25)$ & 5 \\
\hline Jie C & 2017 & China & Ultrasound & Rotterdam & $400(225)$ & $100(38)$ & 5 \\
\hline Kim JJ & 2017 & Korea & Ultrasound & Rotterdam & $275(15)$ & $892(25)$ & 6 \\
\hline
\end{tabular}

AES: Androgen Excess Society criteria NAFLD-LFS: NAFLD liver fat score

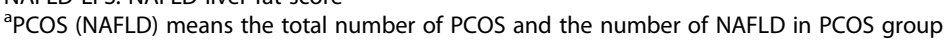

\#control (NAFLD) means the total number of control and the number of NAFLD in control group

${ }^{\mathrm{b}}$ The quality of the included studies was assessed using the Newcastle-Ottawa Scale (NOS) and the score ranged between 0 and 9 
comparing PCOS to controls for risk of NAFLD

Study
ID

Fig. 2 The forest plots show the individual and pooled ORs (95\% Cls) obtained from studies when comparing PCOS to controls for risk of NAFLD

BMI and diagnostic criteria for PCOS or NAFLD. However, there was significant heterogeneity in non-HA studies $\left(I^{2}=83.3 \%\right.$, P-heterogeneity $=0.002$; Table 2$)$.

\section{Sensitivity analysis and publication bias}

Begg's test and Egger's test were used to assess for publication bias for the included studies. There was evidence of publication bias in the overall meta-analysis for risk of NAFLD (Begg's test: $P=0.01$; Egger's test: $P=0.02$ ). Furthermore, the trim-and-fill method developed by Duval and Tweedie was used to adjust for bias [23]. The adjusted result from the fixed model had an OR of 2.08 (95\% CI $=1.81-2.40)$, which was similar to the results of the present study $(\mathrm{OR}=2.25,95 \% \mathrm{CI}=1.95-2.60)$. Thus, the results of the present study are reliable. A sensitivity analysis was further performed by removing one study at a time from the meta-analysis. None of the results were significantly altered when a single study was removed. The pooled OR ranged within 2.18-2.38, confirming the robustness of these results. a

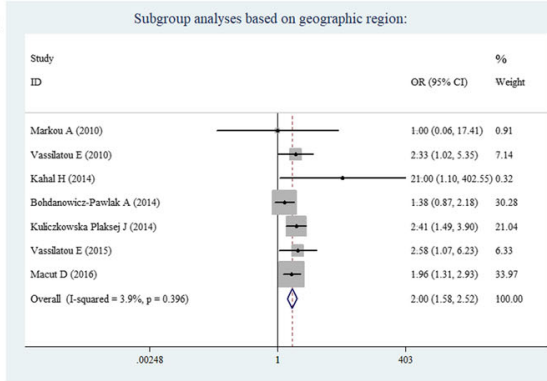

C

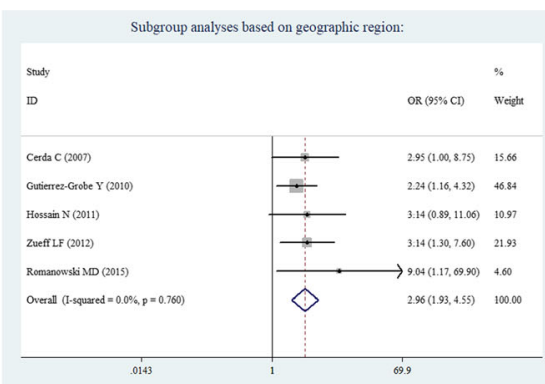

b

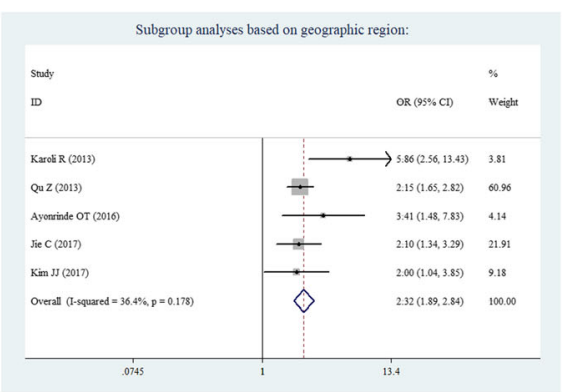

Fig. 3 Subgroup analyses based on geographic region: a Europe, b Asia-Pacific Region, and c America 
a

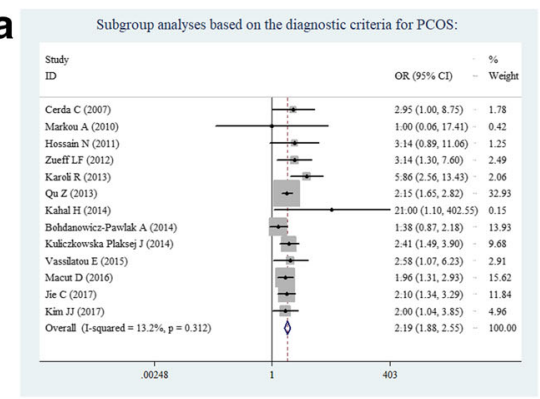

C

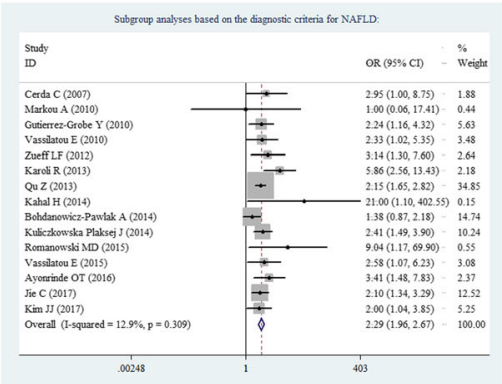

b

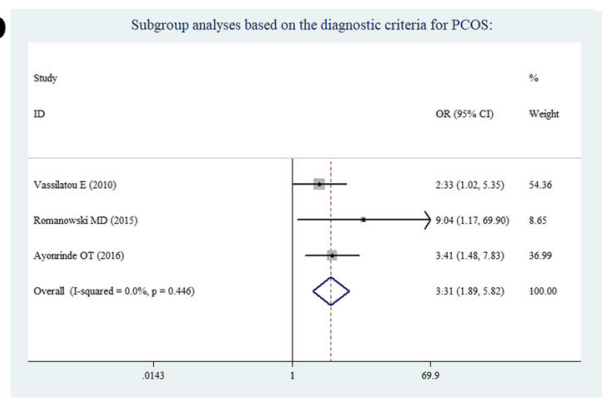

Fig. 4 Subgroup analyses based on the diagnostic criteria for PCOS or NAFLD: a Rotterdam criteria, b no Rotterdam criteria, and c ultrasonography for NAFLD

\section{Discussion}

At present, epidemiological literature regarding the effect of PCOS as a risk factor for NAFLD development remains inconsistent and inconclusive. This controversy needs to be clarified, since the relationship between PCOS and NAFLD is extremely relevant in clinic, both disorders are common, and the coexistence might synergistically increase the risk for both T2DM and cardiovascular disease.

In order to shed some light, a meta-analysis that included seven studies was conducted. It was reported that the prevalence of NAFLD is markedly increased in women with PCOS. Therefore, PCOS has emerged as a significant risk factor for the development of NAFLD
[18]. However, a question that should be addressed is whether NAFLD is associated with PCOS as a consequence of shared risk factors, or whether PCOS contributes to NAFLD in an independent fashion. Therefore, the present meta-analysis was conducted to shed light into the relationship between PCOS and NAFLD. According to the results of the present meta-analysis, women with PCOS had a 2-fold higher chance of developing NAFLD, which was independent of BMI and geographic region. Furthermore, hyperandrogenic women with PCOS had a significantly higher risk of NAFLD than controls. However, non-HA PCOS patients were not associated with the increased prevalence of NAFLD, when compared to controls. This finding suggests that

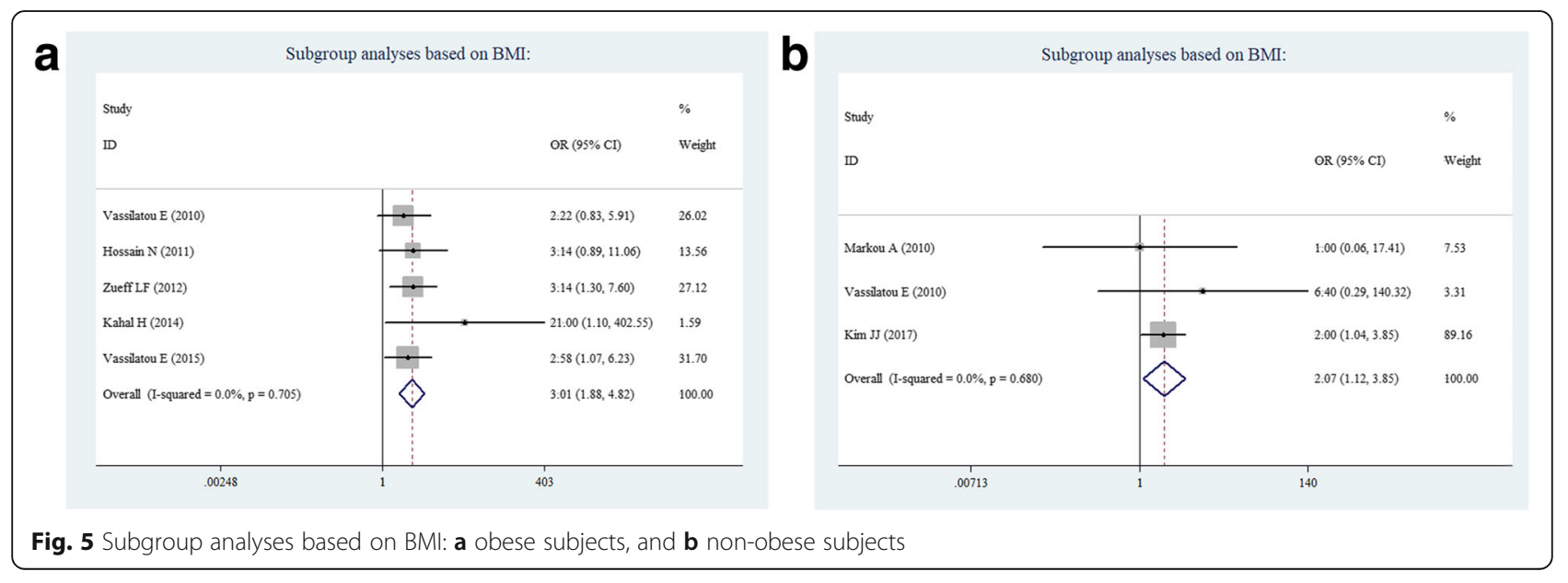



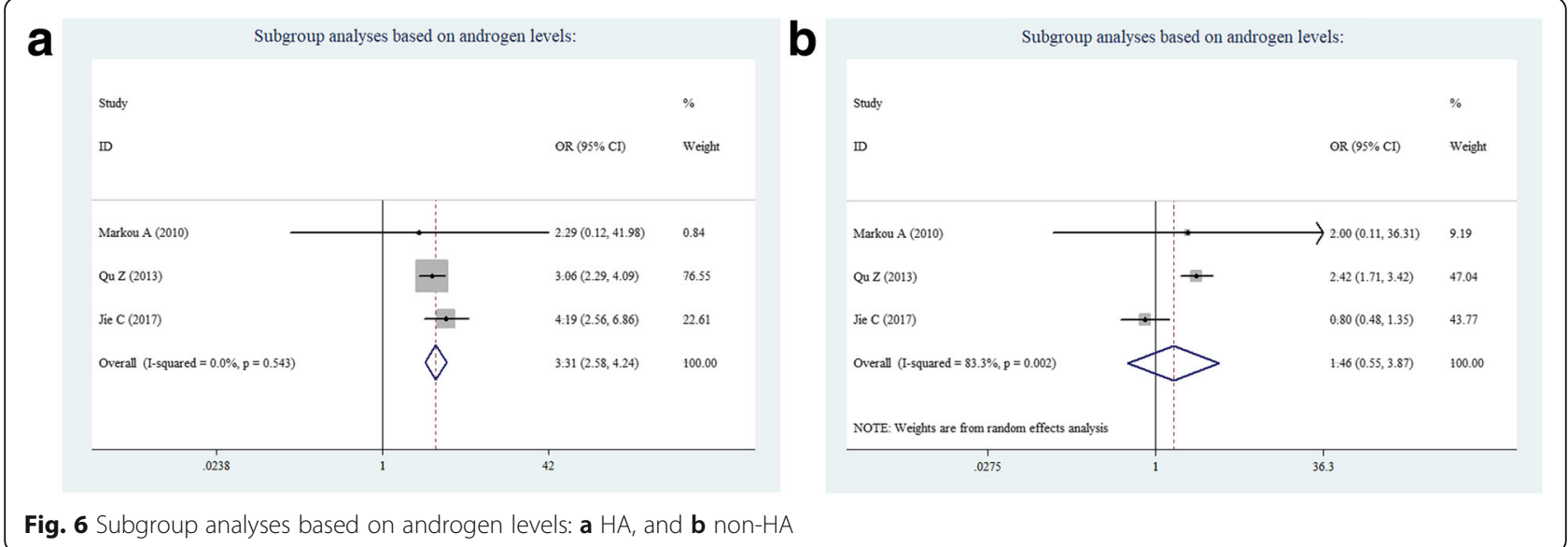

Fig. 6 Subgroup analyses based on androgen levels: a HA, and b non-HA

the putative mechanism linking PCOS and NAFLD is most likely associated with HA.

The association between hyperandrogenic women with PCOS and NAFLD risk is supported by several biological mechanisms. First, androgens can suppress the transcription of the low-density lipoprotein receptor (LDLR) gene, thereby prolonging the half-life of very low-density lipoprotein (VLDL) and LDL. As a result, lipids accumulate in the liver, and hyperandrogenic women with PCOS become more prone to fatty liver [39]. Second, hyperandrogenemia can induce a low-grade inflammatory state by increasing the transcripts of the androgen receptor and the release of TNF- $\alpha$ from mononuclear cells [40], and both contributes to the development of NAFLD [41]. Third, the pathogenesis of NAFLD is multifactorial, but IR appears to be a pivotal contributing factor [7]. Both clinical observations and animal studies have demonstrated that IR could be induced by hyperandrogenemia [42]. Su et al. reported that adult female mice treated with testosterone exhibited IR via the blockade of insulin signal transduction, without influencing body weight and body fat content [43]. Another study conducted by Polderman et al. reported that the testosterone administration to females can induce IR in healthy subjects [44]. Finally, increased androgen levels can promote visceral fat accumulation by inhibiting adenosine monophosphate-activated protein kinase (AMPK) activation, which is a potent inhibitor of lipogenesis in adipose tissues [45]. Visceral obesity is also associated with NAFLD. Thus, an excess in androgen levels may contribute to NAFLD in PCOS patients, affecting the liver directly or indirectly through the modulation of insulin sensitivity, increasing visceral adiposity, or a combination of both.

There were several limitations in the present metaanalysis. First, although the collected publications were all eligible, the studies included in the meta-analysis were limited. Meanwhile, these limited number of studies precluded any meaningful subgroup analyses stratified by important confounding factors such as IR. Second, the method used to assess NAFLD in the majority of studies

Table 2 Stratified analyses for PCOS and risk of NAFLD

\begin{tabular}{|c|c|c|c|c|c|}
\hline & & Studies, n & $\mathrm{OR}^{\mathrm{a}}(95 \% \mathrm{Cl})$ & $1^{2}(\%)$ & Heterogeneity \\
\hline & & & & & 0.40 \\
\hline \multirow[t]{2}{*}{ Diagnostic criteria for PCOS } & Rotterdam criteria & 13 & $2.19(1.88-2.55)$ & 13.2 & 0.312 \\
\hline & no Rotterdam criteria ${ }^{b}$ & 3 & $3.31(1.89-5.82)$ & 0 & 0.446 \\
\hline Diagnosis for NAFLD & ultrasonography & 15 & $2.29(1.96-2.67)$ & 12.9 & 0.309 \\
\hline \multirow[t]{2}{*}{ BMI } & Non-obese subjects ${ }^{c}$ & 3 & $2.07(1.12-3.85)$ & 0 & 0.680 \\
\hline & obese subjects & 5 & $3.01(1.88-4.82)$ & 0 & 0.705 \\
\hline \multirow[t]{2}{*}{ Androgen levels } & $\mathrm{HA}$ & 3 & $3.31(2.58-4.24)$ & 0 & 0.543 \\
\hline & Non- HA & 3 & $1.46(0.55-3.87)$ & 83.3 & 0.002 \\
\hline \multirow[t]{3}{*}{ Geographic region } & Asia-Pacific & 5 & $2.32(1.89-2.84)$ & 36.4 & 0.178 \\
\hline & America & 5 & $2.96(1.93-4.55)$ & 0 & 0.760 \\
\hline & Europe & 7 & $2.00(1.58-2.52)$ & 3.9 & 0.396 \\
\hline
\end{tabular}

${ }^{\mathrm{a}} \mathrm{OR}$ comparing groups of PCOS to the matched controls with regard to the risk of NAFLD

${ }^{b}$ including $\mathrm{NIH}$ criteria or Androgen Excess Society criteria

'defined as $\mathrm{BMI}<25 \mathrm{~kg} / \mathrm{m}^{2}$ 
was ultrasonography, and it has been demonstrated that liver biopsy is the gold standard for the diagnosis of NAFLD. The advantages of ultrasonography include noninvasiveness, reproducibility, low cost, and satisfactory sensitivity and specificity for epidemiologic studies. Third, publication bias existed among the involved studies. However, the trim and fill method was subsequently performed. The OR after adjustment changed to 2.08 , which was almost consistent with the unadjusted result. Thus, the present results are reliable. Fourth, it was found that PCOS without HA was not associated with increased NAFLD risk, when compared to controls. However, few of the included studies had high heterogeneity. Finally, the entire body of evidence was observational, which may be biased due to unmeasured confounders. Although some studies were adjusted for important confounding factors, other modifiable factors were not accounted for in all these studies, such as family history, differences in diet, and/or physical activity patterns. Therefore, residual confounders may be present, which may influence these results. Thus, these observed effects may be attributable to these factors, as opposed to PCOS alone. Conversely, it could not be excluded that confounding factors may mask a more influential factor, leading to the underestimation of the effects of PCOS. Thus, future prospective high-quality studies may be necessary to conclusively address this point.

\section{Conclusion}

In summary, the results of the meta-analysis suggest that PCOS is significantly associated with high risk of NAFLD. This association was dependent of androgen levels, which is the main feature of PCOS, and was interrelated to IR. These findings have clinical implications for NAFLD screening in hyperandrogenic women with PCOS.

\section{Abbreviations}

AES: Androgen Excess Society criteria; Cls: Confidence intervals; HA: Hyperandrogenism; LDLR: Low-density lipoprotein receptor; MS: Metabolic syndrome; NAFLD: Non-alcoholic fatty liver disease; NASH: Nonalcoholic steatohepatitis; ORs: Odds ratios; PCOS: Polycystic ovary syndrome; T2DM: Type-2 diabetes mellitus

\section{Funding}

This study was supported by the Jiangsu Young Medical Talents Project (QNRC2016269), the Applied Basic Research Fund of Changzhou, China (Grant no. CJ20140022), the High-level Medicine Talents Training Project (2016CZBJ022), and the Youth Science and Technology Project of Changzhou Health and Family Planning Commission (QN201601).

\section{Availability of data and materials}

The datasets used and/or analyzed during the present study are available from the corresponding author upon reasonable request.

\section{Authors' contributions}

X-Y.W. and R-X.S. provided the original idea. X-Y.W., X-Y.Y., R-X.S. and J.W carried out the data extraction, analysis and interpretation of data, and drafted the article. S-F.L. carried out the statistical analysis. All authors read and approved the final manuscript.
Ethics approval and consent to participate

Not applicable.

\section{Competing interest}

The authors declare that they have no competing interests.

\section{Publisher's Note}

Springer Nature remains neutral with regard to jurisdictional claims in published maps and institutional affiliations.

\section{Author details}

'Department of Gynecology, Changzhou No. 2 Hospital, Affiliated with Nanjing Medical University, Changzhou 213000, China. ${ }^{2}$ Department of Gastroenterology, Changzhou No. 2 Hospital, Affiliated with Nanjing Medical University, Changzhou 213000, China.

Received: 14 August 2017 Accepted: 29 April 2018

Published online: 10 May 2018

References

1. Kim D, Kim WR. Nonobese fatty liver disease. Clin Gastroenterol Hepatol. 2017;15(4):474-85.

2. Chalasani N, Younossi Z, Lavine JE, Diehl AM, Brunt EM, Cusi K, Charlton M, Sanyal AJ. The diagnosis and management of non-alcoholic fatty liver disease: practice guideline by the American Association for the Study of Liver Diseases, American College of Gastroenterology, and the American Gastroenterological Association. Hepatology. 2012;55(6):2005-23.

3. Doycheva I, Watt KD, Alkhouri N. Nonalcoholic fatty liver disease in adolescents and young adults: the next frontier in the epidemic. Hepatology. 2017;65(6):2100-9.

4. Angulo P. Nonalcoholic fatty liver disease. N Engl J Med. 2002;346(16):1221-31.

5. Makri E, Tziomalos K. Prevalence, etiology and management of nonalcoholic fatty liver disease in patients with polycystic ovary syndrome. Minerva Endocrinol. 2017:42(2):122-31.

6. Clark JM, Diehl AM. Nonalcoholic fatty liver disease: an underrecognized cause of cryptogenic cirrhosis. JAMA. 2003;289:3000-4.

7. Marchesini G, Brizi M, Bianchi G, Tomassetti S, Bugianesi E, Lenzi M, McCullough AJ, Natale S, Forlani G, Melchionda N. Nonalcoholic fatty liver disease: a feature of the metabolic syndrome. Diabetes. 2001;50(8):1844-50.

8. Marchesini G, Brizi M, Morselli-Labate AM, Bianchi G, Bugianesi E, McCullough AJ, Forlani G, Melchionda N. Association of nonalcoholic fatty liver disease with insulin resistance. Am J Med. 1999;107(5):450-5.

9. Bugianesi E, Gastaldelli A, Vanni E, Gambino R, Cassader M, Baldi S, Ponti V, Pagano G, Ferrannini E, Rizzetto M. Insulin resistance in non-diabetic patients with non-alcoholic fatty liver disease: sites and mechanisms. Diabetologia. 2005;48(4):634-42.

10. Goodarzi MO, Dumesic DA, Chazenbalk G, Azziz R. Polycystic ovary syndrome: etiology, pathogenesis and diagnosis. Nat Rev Endocrinol. 2011;7:219-31.

11. Glintborg D, Andersen M. MANAGEMENT OF ENDOCRINE DISEASE: morbidity in polycystic ovary syndrome. Eur J Endocrinol. 2017;176(2):R53-65.

12. Brown AJ, Tendler DA, McMurray RG, Setii TL. Polycystic ovary syndrome and severe nonalcoholic steatohepatitis: beneficial effect of modest weight loss and exercise on liver biopsy findings. Endocr Pract. 2005;11(5):319-24.

13. Jie C, Chunhua W, Yi Z, Yuying W, Wendi X, Tzuchun L, Shengxian L, Lihua W, Jun Z, Yun S, Wei L, Tao T. High free androgen index is associated with increased risk of non-alcoholic fatty liver disease in women with polycystic ovary syndrome, independently of obesity and insulin resistance. Int J Obes (Lond). 2017:41(9):1341-47.

14. Kim JJ, Kim D, Yim JY, Kang JH, Han KH, Kim SM, Hwang KR, Ku SY, Suh CS, Kim SH, Choi YM. Polycystic ovary syndrome with hyperandrogenism as a risk factor for non-obese non-alcoholic fatty liver disease. Aliment Pharmacol Ther. 2017:45(11):1403-12.

15. Macut D, Tziomalos K, Božić-Antić I, Bjekić-Macut J, Katsikis I, Papadakis E, Andrić Z, Panidis D. Non-alcoholic fatty liver disease is associated with insulin resistance and lipid accumulation product in women with polycystic ovary syndrome. Hum Reprod. 2016;31(6):1347-53.

16. Markou A, Androulakis II, Mourmouris C, Tsikkini A, Samara C, Sougioultzis S, Piaditis $\mathrm{G}$, Kaltsas $\mathrm{G}$. Hepatic steatosis in young lean insulin resistant women with polycystic ovary syndrome. Fertil Steril. 2010;93(4):1220-6.

17. Bohdanowicz-Pawlak A, Lenarcik-Kabza A, Brona A, Kuliczkowska-Płaksej J, Łaczmański Ł, Zaleska-Dorobisz U, Milewicz A. Non-alcoholic fatty liver disease in women with polycystic ovary syndrome - clinical and metabolic 
aspects and lipoprotein lipase gene polymorphism. Endokrynol Pol. 2014; 65(6):416-21.

18. Ramezani-Binabaj M, Motalebi M, Karimi-Sari H, Rezaee-Zavareh MS, Alavian SM. Are women with polycystic ovarian syndrome at a high risk of nonalcoholic fatty liver disease; a meta-analysis. Hepat Mon. 2014;14(11):e23235.

19. Moher D, Liberati A, Tetzlaff J, Altman DG; PRISMA Group. Preferred reporting items for systematic reviews and meta-analyses: the PRISMA statement. BMJ. 2009;339:b2535.

20. GA Wells, B Shea, D O'Connell, J Peterson, V Welch, M Losos, P Tugwell. The Newcastle-Ottawa Scale (NOS) for assessing the quality of nonrandomised studies in meta-analyses. http://www.ohri.ca/programs/clinical_ epidemiology/oxford.asp. Accessed 13 Aug 2014

21. Begg CB, Mazumdar M. Operating characteristics of a rank correlation test for publication bias. Biometrics. 1994;50:1088-101.

22. Egger M, Davey Smith G, Schneider M, Minder C. Bias in meta-analysis detected by a simple, graphical test. BMJ. 1997;315:629-34.

23. Duval S, Tweedie R. Trim and fill: a simple funnel-plot-based method of testing and adjusting for publication bias in meta-analysis. Biometrics. 2000;56(2):455-63.

24. Polyzos SA, Goulis DG, Kountouras J, Mintziori G, Chatzis P, Papadakis E, Katsikis I, Panidis D. Non-alcoholic fatty liver disease in women with polycystic ovary syndrome: assessment of non-invasive indices predicting hepatic steatosis and fibrosis. Hormones (Athens). 2014;13(4):519-31.

25. Jones H, Sprung VS, Pugh CJ, Daousi C, Irwin A, Aziz N, Adams VL, Thomas EL, Bell JD, Kemp GJ, Cuthbertson DJ. Polycystic ovary syndrome with hyperandrogenism is characterized by an increased risk of hepatic steatosis compared to nonhyperandrogenic PCOS phenotypes and healthy controls, independent of obesity and insulin resistance. J Clin Endocrinol Metab. 2012;97(10):3709-16.

26. Borruel $\mathrm{S}$, Fernández-Durán E, Alpañés M, Martí D, Alvarez-Blasco F, LuqueRamírez M, Escobar-Morreale HF. Global adiposity and thickness of intraperitoneal and mesenteric adipose tissue depots are increased in women with polycystic ovary syndrome (PCOS). J Clin Endocrinol Metab. 2013;98(3):1254-63.

27. Cerda C, Pérez-Ayuso RM, Riquelme A, Soza A, Villaseca P, Sir-Petermann T, Espinoza M, Pizarro M, Solis N, Miquel JF, Arrese M. Nonalcoholic fatty liver disease in women with polycystic ovary syndrome. J Hepatol. 2007;47(3):412-7.

28. Gutierrez-Grobe $Y$, Ponciano-Rodríguez G, Ramos MH, Uribe M, MéndezSánchez N. Prevalence of non-alcoholic fatty liver disease in premenopausal, postmenopausal and polycystic ovary syndrome women. The role of estrogens. Ann Hepatol. 2010;9(4):402-9.

29. Vassilatou E, Lafoyianni S, Vryonidou A, loannidis D, Kosma L, Katsoulis K, Papavassiliou E, Tzavara I. Increased androgen bioavailability is associated with non-alcoholic fatty liver disease in women with polycystic ovary syndrome. Hum Reprod. 2010;25(1):212-20.

30. Hossain N, Stepanova M, Afendy A, Nader F, Younossi Y, Rafiq N, Goodman Z, Younossi ZM. Non-alcoholic steatohepatitis (NASH) in patients with polycystic ovarian syndrome (PCOS). Scand I Gastroenterol. 2011;46(4):479-84.

31. Zueff LF, Martins WP, Vieira CS, Ferriani RA. Ultrasonographic and laboratory markers of metabolic and cardiovascular disease risk in obese women with polycystic ovary syndrome. Ultrasound Obstet Gynecol. 2012;39(3):341-7.

32. Karoli R, Fatima J, Chandra A, Gupta U, Islam FU, Singh G. Prevalence of hepatic steatosis in women with polycystic ovary syndrome. J Hum Reprod Sci. 2013;6(1):9-14

33. Qu Z, Zhu Y, Jiang J, Shi Y, Chen Z. The clinical characteristics and etiological study of nonalcoholic fatty liver disease in Chinese women with PCOS. Iran J Reprod Med. 2013;11(9):725-32.

34. Kahal H, Abouda G, Rigby AS, Coady AM, Kilpatrick ES, Atkin SL. Glucagonlike peptide-1 analogue, liraglutide, improves liver fibrosis markers in obese women with polycystic ovary syndrome and nonalcoholic fatty liver disease. Clin Endocrinol. 2014;81(4):523-8

35. Kuliczkowska Plaksej J, Laczmanski L, Milewicz A, Lenarcik-Kabza A, TrzmielBira A, Zaleska-Dorobisz U, Lwow F, Hirnle L. Cannabinoid receptor 1 gene polymorphisms and nonalcoholic fatty liver disease in women with polycystic ovary syndrome and in healthy controls. Int J Endocrinol. 2014;2014:232975.

36. Romanowski MD, Parolin MB, Freitas AC, Piazza MJ, Basso J, Urbanetz AA Prevalence of non-alcoholic fatty liver disease in women with polycystic ovary syndrome and its correlation with metabolic syndrome. Arq Gastroenterol. 2015;52(2):117-23.

37. Vassilatou E, Vassiliadi DA, Salambasis K, Lazaridou H, Koutsomitopoulos $N$, Kelekis N, Kassanos D, Hadjidakis D, Dimitriadis G. Increased prevalence of polycystic ovary syndrome in premenopausal women with nonalcoholic fatty liver disease. Eur J Endocrinol. 2015;173(6):739-47.

38. Ayonrinde OT, Adams LA, Doherty DA, Mori TA, Beilin LJ, Oddy WH, Hickey M, Sloboda DM, Olynyk JK, Hart R. Adverse metabolic phenotype of adolescent girls with non-alcoholic fatty liver disease plus polycystic ovary syndrome compared with other girls and boys. J Gastroenterol Hepatol. 2016;31(5):980-7.

39. Baranova A, Tran TP, Afendy A, Wang L, Shamsaddini A, Mehta R, Chandhoke V, Birerdinc A, Younossi ZM. Molecular signature of adipose tissue in patients with both non-alcoholic fatty liver disease (NAFLD) and polycystic ovarian syndrome (PCOS). J Transl Med. 2013;11:133.

40. González F, Sia CL, Bearson DM, Blair HE. Hyperandrogenism induces a proinflammatory TNFa response to glucose ingestion in a receptordependent fashion. J Clin Endocrinol Metab. 2014;99(5):E848-54.

41. Alisi A, Carpino G, Oliveira FL, Panera N, Nobili V, Gaudio E. The role of tissue macrophage-mediated inflammation on NAFLD pathogenesis and its clinical implications. Mediat Inflamm. 2017;2017:8162421.

42. Livingstone C, Collison M. Sex steroids and insulin resistance. Clin Sci (Lond). 2002;102(2):151-66.

43. Su CL, Chen M, Xu W, Lin JF. The impacts of testosterone on insulin sensitivity and chronic low-grade. Zhonghua Yi Xue Za Zhi. 2017;97(1):47-52

44. Polderman KH, Gooren LJ, Asscheman H, Bakker A, Heine RJ. Induction of insulin resistance by androgens and estrogens. J Clin Endocrinol Metab. 1994;79(1):265-71.

45. McInnes KJ, Corbould A, Simpson ER, Jones ME. Regulation of adenosine 5', monophosphate-activated protein kinase and lipogenesis by androgens contributes to visceral obesity in an estrogen-deficient state. Endocrinology. 2006;147(12):5907-13.

\section{Ready to submit your research? Choose BMC and benefit from:}

- fast, convenient online submission

- thorough peer review by experienced researchers in your field

- rapid publication on acceptance

- support for research data, including large and complex data types

- gold Open Access which fosters wider collaboration and increased citations

- maximum visibility for your research: over $100 \mathrm{M}$ website views per year

At BMC, research is always in progress.

Learn more biomedcentral.com/submissions 